\title{
BLOW-UP FOR SIGN-CHANGING SOLUTIONS OF THE CRITICAL HEAT EQUATION IN DOMAINS WITH A SMALL HOLE
}

\author{
ISABELLA IANNI, MONICA MUSSO, AND ANGELA PISTOIA
}

Abstract. We consider the critical heat equation

$$
\begin{array}{lr}
v_{t}-\Delta v=|v|^{\frac{4}{n-2}} v & \Omega_{\epsilon} \times(0,+\infty) \\
v=0 & \partial \Omega_{\epsilon} \times(0,+\infty) \\
v=v_{0} & \text { in } \Omega_{\epsilon} \times\{t=0\}
\end{array}
$$

in $\Omega_{\epsilon}:=\Omega \backslash B_{\epsilon}\left(x_{0}\right)$ where $\Omega$ is a smooth bounded domain in $\mathbb{R}^{N}, N \geq 3$ and $B_{\epsilon}\left(x_{0}\right)$ is a ball of $\mathbb{R}^{N}$ of center $x_{0} \in \Omega$ and radius $\epsilon>0$ small.

We show that if $\epsilon>0$ is small enough, then there exists a sign-changing stationary solution $\phi_{\epsilon}$ of $(\mathrm{CH})$ such that the solution of $(\mathrm{CH})$ with initial value $v_{0}=\lambda \phi_{\epsilon}$ blows up in finite time if $|\lambda-1|>0$ is sufficiently small.

This shows in particular that the set of the initial conditions for which the solution of $(\mathrm{CH})$ is global and bounded is not star-shaped.

\section{INTRODUCTION}

We consider the semilinear parabolic equation

$$
\begin{cases}v_{t}-\Delta v=|v|^{p-1} v & \text { in } D \times(0, T) \\ v=0 & \text { on } \partial D \times(0, T) \\ v(0)=v_{0} & \text { in } D\end{cases}
$$

where $D$ is a smooth bounded domain in $\mathbb{R}^{N}, N \in \mathbb{N}, N \geq 3$ and $p>1$.

For any $p>1$ problem (1.1) is locally well-posed for $v_{0} \in C_{0}(D)$, where

$$
C_{0}(D)=\{v \in C(\bar{D}), v=0 \text { on } \partial D\} .
$$

Let $T_{\max }\left(v_{0}\right) \in(0,+\infty]$ denote the maximal existence time of the unique local in time classical solution $v=v(\cdot, t)$ of $(1.1)$.

The solution $v$ is said to be global when $T_{\max }\left(v_{0}\right)=\infty$, while when $T_{\max }\left(v_{0}\right)<\infty$ it is said to blow-up in finite time.

Let us define the set of initial values such that the solution is global

$$
\mathcal{G}=\left\{v_{0} \in C_{0}(D), T_{\max }\left(v_{0}\right)=+\infty\right\}
$$

Date: July 11, 2021.

2000 Mathematics Subject Classification. 35K91, 35B35, 35B44, 35J91.

Key words and phrases. Semi-linear parabolic equations, sign-changing stationary solutions, critical Sobolev exponent, blow-up phenomena.

${ }^{0}$ The second author has been supported by Fondecyt Grant 1120151. The first and the third authors have been partially supported by the Gruppo Nazionale per l'Analisi Matematica, la Probabilitá e le loro Applicazioni (GNAMPA) of the Istituto Nazionale di Alta Matematica (INdAM) and PRIN 2012 grant(Italy). 
and its complementary set of initial conditions for which the corresponding solution blows-up in finite time

$$
\mathcal{F}=\left\{v_{0} \in C_{0}(D), T_{\max }\left(v_{0}\right)<+\infty\right\} .
$$

Let also $\mathcal{B} \subseteq \mathcal{G}$ be the set of initial values for which the solution is global in time and has an $L^{\infty}$-global bound

$$
\mathcal{B}=\left\{v_{0} \in C_{0}(D), T_{\max }\left(v_{0}\right)=+\infty \text { and } \sup _{t \geq 0}\|v(t)\|_{L^{\infty}}<\infty\right\}
$$

When $p$ is subcritical, namely $1<p<p_{S}$ where

$$
p_{S}=\frac{N+2}{N-2}
$$

$\left(2^{*}=p_{S}+1\right.$ is the critical Sobolev exponent $)$, one has that $\mathcal{B}=\mathcal{G}$ since

$$
T_{\max }\left(v_{0}\right)=+\infty \Rightarrow \sup _{t \geq 0}\|v(t)\|_{L^{\infty}}<\infty
$$

but for $p \geq p_{S}$ it may occur that $\mathcal{B} \subset \mathcal{G}$. In the critical case $p=p_{S}$ for instance, it is well known that infinite time blow-up may occur, namely there may exist $v_{0} \in C_{0}(D)$ such that

$$
T_{\max }\left(v_{0}\right)=+\infty \text { and } \lim _{t \uparrow+\infty}\|v(t)\|_{L^{\infty}}=+\infty .
$$

(cfr. [6,7,15] for a radial positive $v_{0} \in \mathcal{G} \backslash \mathcal{B}$ when $D$ is a ball, and [18] for a positive $v_{0} \in \mathcal{G} \backslash \mathcal{B}$ when $D$ is convex and symmetric, see also [10] for necessary and sufficient conditions for the $L^{\infty}$ global bound in the critical and subcritical case).

Let us observe that all the stationary solutions of (1.1) belong to $\mathcal{B}$ (if any: when $p \geq p_{S}$ and $D$ is star-shaped for instance the only stationary solution is the trivial one, cfr. [16]), moreover $\mathcal{B}$ contains a neighborhood of the origin (since the zero solution is exponentially asymptotically stable in $L^{\infty}$, see [17, Theorem 19.2]).

If we restrict ourselves to non-negative initial data, then the solutions are positive by the parabolic maximum principle, hence we may replace the nonlinearity $|v|^{p-1} v$ by $|v|^{p}$ which is convex and so (see [12]) the corresponding sets $\mathcal{G}^{+}=\left\{v_{0} \in \mathcal{G}, v_{0} \geq 0\right\}$ and $\mathcal{B}^{+}=\left\{v_{0} \in \mathcal{B}, v_{0} \geq 0\right\}$ are convex, hence star-shaped around 0 .

More specifically if $\phi$ is a stationary positive solution to (1.1) and $v_{0}=\lambda \phi$ for $\lambda>0$, then $v_{0}>\phi$ if $\lambda>1$ and so $v_{0} \in \mathcal{F}$ (see for instance [17, Theorem 17.8]), while $0 \leq v_{0} \leq \phi$ for $0<\lambda \leq 1$ and so $v_{0} \in \mathcal{B}$ (since by the parabolic maximum principle $0<v(t) \leq \phi$ for $t>0$ and moreover [17, Lemma 17.9] applies).

If we consider sign-changing initial data then the arguments above can not be applied. In particular if $\phi$ is a sign-changing stationary solution to (1.1), then it is not comparable with $\lambda \phi$ for $\lambda \neq 1$. Anyway, since the zero solution is exponentially asymptotically stable in $L^{\infty}$, then clearly $\lambda \phi \in \mathcal{B}$ for $\lambda$ sufficiently small, moreover it is also known that $\lambda \phi \in \mathcal{F}$ for $\lambda$ sufficiently large (see [17, Theorem 17.6]) and of course if $\lambda=1$ then $\lambda \phi \in \mathcal{B}$.

Recently it has been proved (cfr. [2] when $D$ is a ball, [13] for any smooth bounded domain $D)$, that if $p$ is subcritical and sufficiently close to the critical exponent, then there exist sign-changing stationary solutions $\phi$ such that $\lambda \phi \in \mathcal{F}$ for $\lambda>0$ sufficiently close to 1 . This 
results shows that in the subcritical case $\mathcal{B}$ in general is not star-shaped around 0 , hence not convex.

The aim of this paper is to extend this result to the critical case $p=p_{S}$.

For $p=p_{S}$ already the existence of a (sign-changing) stationary solution $\phi$ of (1.1) is an issue. Indeed it is well known that there are no nontrivial stationary solutions when $D$ is strictly starshaped (cfr. [16]), while it is easy to prove the existence of infinitely many radial stationary solutions if $D$ is an annulus (cfr. [11]). It is also known that there is a positive stationary solution whenever the homology of dimension $d$ of $D$ with $\mathbb{Z}_{2}$ coefficients is nontrivial for some positive integer $d$ (cfr. [1]).

We consider here problem (1.1) when $p=p_{S}$ and $D$ is a domain with a small hole, precisely

$$
D:=\Omega_{\epsilon}=\Omega \backslash B_{\epsilon}\left(x_{0}\right),
$$

where $\Omega$ is a smooth bounded domain in $\mathbb{R}^{N}, N \in \mathbb{N}, N \geq 3$ and $B_{\epsilon}\left(x_{0}\right) \subset \Omega$ is a ball of $\mathbb{R}^{N}$ of center $x_{0} \in \Omega$ and radius $\epsilon>0$ small enough.

Under these assumptions the existence of a positive stationary solution is a classical result by Coron (cfr. [3]), while the existence of an arbitrary large number of sign-changing stationary solutions has been obtained in more recent works by Musso and Pistoia (cfr. [14]) and Ge, Musso and Pistoia (cfr. [9]), in the case when the hole is small enough.

Our main result is the following

Theorem 1.1. Let $\Omega_{\epsilon}:=\Omega \backslash B_{\epsilon}\left(x_{0}\right)$ where $\Omega$ is a smooth bounded domain in $\mathbb{R}^{N}, N \in \mathbb{N}$, $N \geq 3, x_{0} \in \Omega$ and $\epsilon>0$ small.

There exists $\epsilon_{0}>0$ with the following property. If $0<\epsilon \leq \epsilon_{0}$ then there exists a signchanging stationary solution $\phi_{\epsilon}$ of

$$
\begin{cases}v_{t}-\Delta v=|v|^{\frac{4}{N-2}} v & \text { in } \Omega_{\epsilon} \times(0, T) \\ v=0 & \text { on } \partial \Omega_{\epsilon} \times(0, T) \\ v(0)=v_{0} & \text { in } \Omega_{\epsilon}\end{cases}
$$

and a constant $\delta_{\epsilon}>0$ such that if $\lambda>0,0<|\lambda-1|<\delta_{\epsilon}$ then the classical solution $v$ of (1.4) with initial value $v_{0}=\lambda \phi_{\epsilon}$ blows up, namely $v_{0} \in \mathcal{F} \cup(\mathcal{G} \backslash \mathcal{B})$.

As a consequence $\mathcal{B}$ is not star-shaped around zero.

Observe that now, unlike the subcritical case, the solution $v$ may blow-up in finite or infinite time and so we can not conclude that also the set $\mathcal{G}$ is not convex, unless we restrict to more specific situations like in the following result related to sign-changing radial solutions in an annulus

Theorem 1.2. Let $\Omega_{\epsilon}:=\left\{x \in \mathbb{R}^{N}: 0<\epsilon<|x|<1\right\}, N \in \mathbb{N}, N \geq 3, \epsilon>0$ small.

There exists $\epsilon_{0}>0$ with the following property. If $0<\epsilon \leq \epsilon_{0}$ then there exists a signchanging radial stationary solution $\widehat{\phi}_{\epsilon}$ of (1.4) and a constant $\delta_{\epsilon}>0$ such that if $\lambda>0$, $0<|\lambda-1|<\delta_{\epsilon}$ then the classical solution $v$ of (1.4) with initial value $v_{0}=\lambda \widehat{\phi_{\epsilon}}$ blows-up 
in finite time, namely $v_{0} \in \mathcal{F}$.

As a consequence both $\mathcal{B}$ and $\mathcal{G}$ are not star-shaped around zero.

The sign-changing stationary solution $\phi_{\epsilon}$ of Theorem 1.1 is any bubble tower solution found in [9]. The proof consists then in scaling it properly and performing an asymptotic spectral analysis of the linearized problem, similarly as it has been done in the almost critical case $([2,13]$, see also $[4,5]$ for the case $N=2)$. Now the exponent of the nonlinearity is fixed, and the scaling parameter depends only on the radius $\epsilon$ of the hole. The asymptotic analysis is possible again thanks to the knowledge of the limit problem. Combining it with general results for the heat flow (see Proposition 2.2 in Section 2), we can show that $\phi_{\epsilon}$ can be compared with the solution $v$ at a certain time $t_{0}>0$. The blow-up result then follows from a blow-up criterion via comparison for sign changing solutions of the critical heat equation (Proposition 2.1).

The proof of Theorem 1.2 is obtained by repeating similar arguments but starting from any of the sign-changing radial bubble tower stationary solutions $\widehat{\phi}_{\epsilon}$ found in [14]. With this choice the solution $v$ is radial and so if it is global then it must satisfy an $L^{\infty}$ global bound (cfr. [10]), thus excluding infinite time blow-up.

\section{Preliminaries}

In this section we provide a blow-up criterion via comparison for sign-changing solutions of the critical heat equation. It extends to the critical case the analogous result already known for the subcritical heat equation (see [2, Proposition B.1] and [8, Theorem 10]). Unlike the subcritical case, both finite time blow-up and infinite time blow up can in general occur now.

Proposition 2.1. Let $\psi \in C_{0}(D)$ be a sign-changing stationary solution of (1.1) with $p=p_{S}$. Let $v_{0} \in C_{0}(D), v_{0} \not \equiv \psi$ be either $v_{0} \geq \psi$ or $v_{0} \leq \psi$, then $v_{0} \in \mathcal{F} \cup(\mathcal{G} \backslash \mathcal{B})$.

If in particular $D$ is an annulus $\left\{x \in \mathbb{R}^{N}: a<|x|<b\right\}$ (for $b>a>0$ ) and $v_{0}$ is radially symmetric, then $v_{0} \in \mathcal{F}$.

Proof. Once the first part is proved, the last assertion follows directly from [10], where it has been showed that a necessary and sufficient condition to get an $L^{\infty}$ global bound for a global in time solution $v$ of $(1.1)$ with $p \in\left(1, p_{S}\right]$ is that the energy functional

$$
J_{p}(u)=\frac{1}{2}\|\nabla u\|_{L^{2}(D)}^{2}-\frac{1}{p+1}\|u\|_{L^{p+1}(D)}^{p+1}, \quad u \in H_{0}^{1}(D)
$$

satifies the Palais-Smale condition along $v$. Indeed when the domain $D$ in an annulus and $v_{0}$ is radial then the solution $v$ of $(1.1)$ with initial condition $v(0)=v_{0}$ is radial and the Sobolev compact embedding $H_{0, r}^{1}(D) \subset \subset L^{2^{*}}(D)$ (where $H_{0, r}^{1}(D)$ is the subspace of the radial functions in $H_{0}^{1}(D)$ ) ensures that $J_{p_{S}}$ satisfies the Palais-Smale condition along $v$.

Next we prove the first part. The proof follows closely the one for the subcritical case, the main difference being now the lack of a priori bounds for the $L^{\infty}$ norm of global solutions. 
We repeat it in details for the reader convenience. We prove the case $v_{0} \geq \psi$, the other case being similar.

Let $v$ be the solution of (1.1) with initial condition $v(0)=v_{0}$. Arguing by contradiction, assume that $v$ doesn't blow-up, namely that

$$
T_{\max }\left(v_{0}\right)=+\infty \quad \text { and } \quad \sup _{t \geq 0}\|v(t)\|_{L^{\infty}}<\infty .
$$

By the parabolic strong comparison principle

$$
v(x, t)>\psi(x), \quad x \in D, t>0,
$$

hence, at time $t=1$, there exists $\epsilon_{0}>0$ such that

$$
v(x, 1)>\psi(x)+\epsilon \varphi_{1}(x), \quad x \in D, 0<\epsilon<\epsilon_{0},
$$

where $\varphi_{1}$ is the first eigenfunction, $\varphi_{1}>0$, normalized by $\left\|\varphi_{1}\right\|_{L^{\infty}}=1$, of the linear operator $-\Delta-p_{S}|\psi(x)|^{p_{S}-1}$ in $D$ with Dirichlet boundary condition.

Let $w$ be the solution of (1.1) with initial condition $w(x, 0)=\psi(x)+\epsilon \varphi_{1}(x), x \in D$. Since $w(x, 0)<v(x, 1)$, by the weak comparison principle it follows that

$$
w(x, t) \leq v(x, t+1), \quad t \geq 0, x \in D .
$$

Moreover, since $\psi<w(0)$ in $D$, by the weak comparison principle

$$
\psi(x) \leq w(x, t), \quad t \geq 0, x \in D .
$$

By (2.1) and (2.2) it then follows that $w$ is global and also that $\|w(t)\|_{L^{\infty}} \leq \max \left\{\|\psi\|_{L^{\infty}}, \| v(t+\right.$ 1) $\left.\|_{L^{\infty}}\right\}, t \geq 0$, and so that

$$
\sup _{t \geq 0}\|w(t)\|_{L^{\infty}}<\infty .
$$

Since the function $w(x, 0)=\psi(x)+\epsilon \varphi_{1}(x)$, for $\epsilon>0$ sufficiently small, is a stationary subsolution for (1.1) (see [2, Lemma B.4]), it follows (cfr. [17, Proposition 52.19]) that $w_{t} \geq 0$ for $x \in D, t \geq 0$, namely $t \mapsto w(t)$ is monotone increasing.

By (2.3) and the monotonicity it follows that there exists $\psi^{\prime} \in C_{0}(D)$, stationary solution of (1.1), such that

$$
w(t) \uparrow \psi^{\prime} \text { in } C_{0}(D) \text {, as } t \rightarrow+\infty .
$$

But then by the monotonicity, recalling that $\varphi_{1} \geq 0$

$$
\psi^{\prime}(x) \geq w(x, t) \geq w(x, 0)=\psi(x)+\epsilon \varphi_{1}(x) \geq \psi(x), \quad x \in D, t>0
$$

from which it follows that $\psi^{\prime} \not \equiv 0$.

Moreover, by the parabolic strong comparison principle,

$$
\psi^{\prime}(x)>w(x, t), \quad x \in D, t>0,
$$

indeed by $(2.4) \psi^{\prime}(x) \geq w(x, 0)$ and moreover $\psi^{\prime}(x) \not \equiv w(x, 0)$ (otherwise $\psi(x)+\epsilon \varphi_{1}(x)$ would be a stationary solution to (1.1), which is not the case).

Hence, at time $t=1$, there exists $\epsilon_{0}^{\prime}>0$ such that

$$
\psi^{\prime}(x)-\epsilon^{\prime} \varphi_{1}^{\prime}(x) \geq w(x, 1), \quad x \in D, 0<\epsilon^{\prime}<\epsilon_{0}^{\prime},
$$

where $\varphi_{1}^{\prime}$ is the first eigenfunction, $\varphi_{1}^{\prime}>0$, normalized by $\left\|\varphi_{1}^{\prime}\right\|_{L^{\infty}}=1$, of the linear operator $-\Delta-p_{S}\left|\psi^{\prime}(x)\right|^{p_{S}-1}$ in $D$ with Dirichlet boundary condition. 
Since for $\epsilon^{\prime}>0$ small enough the function $\psi^{\prime}(x)-\epsilon^{\prime} \varphi_{1}^{\prime}(x)$ is a stationary supersolution for (1.1) (see [2, Lemma B.4]), by the comparison principle we get

$$
\psi^{\prime}(x)-\epsilon^{\prime} \varphi_{1}^{\prime}(x) \geq w(x, t) \quad x \in D, t \geq 1
$$

and passing to the limit as $t \rightarrow+\infty$ we get a contradiction.

In order to prove Theorem 1.1 and Theorem 1.2 we will need the following general result, whose proof can be found in [2]:

Proposition 2.2. Let $\phi \in C_{0}(D)$ be a sign changing stationary solution of (1.1) and let $\varphi_{1}$ be the positive eigenfunction of the self-adjoint operator $L$ given by $L \varphi=-\Delta \varphi-p|\phi|^{p-1} \varphi$, for $\varphi \in H^{2}(D) \cap H_{0}^{1}(D)$. For $\lambda>0$, let $v^{\lambda}$ be the solution of (1.1) with the initial condition $v^{\lambda}(0)=\lambda \phi$. Assume that

$$
\int_{D} \phi \varphi_{1}>0
$$

Then there exist $t_{0}>0$ and $\delta>0$ such that

$$
\begin{array}{ll}
v^{\lambda}\left(t_{0}\right)>\phi, & \text { for } \lambda \in(1,1+\delta], \\
v^{\lambda}\left(t_{0}\right)<\phi, & \text { for } \lambda \in[1-\delta, 1) .
\end{array}
$$

Proof. The proof consists in linearizing the equation (1.1) in $\phi$, by setting $z^{\lambda}$ through

$$
(\lambda-1) z^{\lambda}(t)=u^{\lambda}(t)-\phi,
$$

and than, by means of condition (2.5) and the properties of linear equations, in showing the existence of $t_{0}>0$ and $\delta>0$, such that

$$
z^{\lambda}\left(x, t_{0}\right)>0, \text { for }|\lambda-1| \leq \delta .
$$

We refer the reader to [2] for all the details.

\section{Proof of Theorem 1.1}

The strategy of the proof of Theorem 1.1 is similar to the one in $[2,13]$ : we show the existence of a sign-changing stationary solution to (1.4) which satisfies the assumption (2.5). Then Proposition 2.2 applies and the conclusion follows from a comparison argument.

In our case the comparison result is given by Proposition 2.1.

As the sign-changing stationary solution we take the $k$-tower solution $\phi_{\epsilon}$ built in [9] in domains with a sufficiently small hole (Lemma 3.1 below). Hence the core of the proof will be to show that a $k$-tower stationary solution satisfies the assumption (2.5), this is obtained by an asymptotic spectral analysis of the linearized operator $-\Delta-p_{S}\left|\phi_{\epsilon}\right|^{p_{S}-1}$ as $\epsilon$ goes to zero, and it is the result in Proposition 3.2 below. 
Before stating our results, we need to fix some notation.

Let

$$
U_{\delta, \xi}(x):=\alpha_{N}\left(\frac{\delta}{\delta^{2}+|x-\xi|^{2}}\right)^{\frac{N-2}{2}}, x \in \mathbb{R}^{N}
$$

where $\alpha_{N}:=[N(N-2)]^{\frac{N-2}{4}}, \delta$ is any positive parameter and $\xi$ a point in $\mathbb{R}^{N}$. These functions are the only positive bounded solutions of the critical problem on the whole space

$$
\Delta u+u^{p_{S}}=0 \text { in } \mathbb{R}^{N} .
$$

Lemma 3.1 (Existence of $k$-tower stationary solutions). Let $\Omega_{\epsilon}:=\Omega \backslash B_{\epsilon}\left(x_{0}\right)$ where $\Omega$ is a smooth bounded domain in $\mathbb{R}^{N}, N \in \mathbb{N}, N \geq 3, x_{0} \in \Omega$ and $\epsilon>0$ small. For any integer $k \geq 2$ there exists $\epsilon_{k}>0$ such that for any $\epsilon \in\left(0, \epsilon_{k}\right)$ problem (1.4) has a $k$-tower sign-changing stationary solution $\phi_{\epsilon}$ whose profile is

$$
\phi_{\epsilon}(x)=\sum_{i=1}^{k}(-1)^{i} U_{\delta_{i_{\epsilon}}, \xi_{i \epsilon}}(x)+R_{\epsilon}(x), x \in \Omega_{\epsilon}
$$

where the concentration parameters $\delta_{i \epsilon}$ 's satisfy

$$
\delta_{i \epsilon}:=d_{i \epsilon} \epsilon^{\frac{2 i-1}{2 k}}, d_{i_{\epsilon}} \in \mathbb{R} \quad \text { and } \quad d_{i_{\epsilon}} \rightarrow d_{i}>0 \text { as } \epsilon \rightarrow 0 \quad \text { for } i=1, \ldots, k,
$$

the concentration points $\xi_{i_{\epsilon}}$ 's satisfy

$$
\xi_{i_{\epsilon}}:=x_{0}+\delta_{i \epsilon} \tau_{i \epsilon}, \quad \tau_{i \epsilon} \in \mathbb{R}^{N} \quad \text { and } \quad \tau_{i \epsilon} \rightarrow \tau_{i} \text { as } \epsilon \rightarrow 0 \quad \text { for } i=1, \ldots, k,
$$

and the remainder term $R_{\epsilon}$ satisfies

$$
\left\|R_{\epsilon}\right\|_{L^{\frac{2 N}{N-2}}\left(\Omega_{\epsilon}\right)} \rightarrow 0 \text { as } \epsilon \rightarrow 0 .
$$

Proof. In [9] it was proved that for any integer $k \geq 1$ there exists $\epsilon_{k}>0$ such that for any $\epsilon \in\left(0, \epsilon_{k}\right)$ problem (1.4) has a stationary solution $\phi_{\epsilon}$ whose profile is

$$
\phi_{\epsilon}(x)=\sum_{i=1}^{k}(-1)^{i} P_{\Omega_{\epsilon}} U_{\delta_{i \epsilon}, \xi_{i \epsilon}}(x)+\psi_{\epsilon}(x), x \in \Omega_{\epsilon}
$$

where the concentration parameters $\delta_{1}=\delta_{1 \epsilon}, \ldots, \delta_{k}=\delta_{k \epsilon}$ satisfy (3.4) and the concentration points $\xi_{1}=\xi_{1_{\epsilon}}, \ldots, \xi_{k}=\xi_{k_{\epsilon}}$ satisfy (3.5). Here $P_{\Omega_{\epsilon}} U_{\delta_{i}, \xi_{i}}$ denotes the projection of the bubble $U_{\delta_{i}, \xi_{i}}$ onto $H_{0}^{1}\left(\Omega_{\epsilon}\right)$, namely the solution of

$$
\Delta P_{\Omega_{\epsilon}} U_{\delta_{i}, \xi_{i}}=\Delta U_{\delta_{i}, \xi_{i}} \text { in } \Omega_{\epsilon}, \quad P_{\Omega_{\epsilon}} U_{\delta_{i}, \xi_{i}}=0 \text { on } \partial \Omega_{\epsilon} .
$$

Moreover, the remainder term $\psi_{\epsilon}$ satisfies (see, for example, Proposition 2.1 in [9])

$$
\left\|\psi_{\epsilon}\right\|_{H_{0}^{1}\left(\Omega_{\epsilon}\right)} \rightarrow 0 \text { as } \epsilon \rightarrow 0 .
$$

It is important to point out that the projection of the bubble has the following expansion (see, for example, Lemma 3.1 in [9])

$$
P_{\Omega_{\epsilon}} U_{\delta_{i}, \xi_{i}}(x)=U_{\delta_{i}, \xi_{i}}(x)-\alpha_{N} \delta_{i}^{\frac{N-2}{2}} H\left(x, x_{0}\right)-\alpha_{N} \frac{\epsilon^{N-2}}{\delta_{i}^{\frac{N-2}{2}}\left(1+\left|\tau_{i}\right|^{2}\right)^{\frac{N-2}{2}}} \frac{1}{\left|x-x_{0}\right|^{N-2}}+R_{i_{\epsilon}}(x),
$$


where the function $H(x, y)$ in (3.9) is the regular part of the Green function $G(x, y)$ of the Laplace operator in $\Omega$ with zero Dirichlet boundary condition, and $R_{i \epsilon}$ satisfies the pointwise estimate

$$
\left|R_{i \epsilon}(x)\right| \leq c \delta_{i}^{\frac{N-2}{2}}\left[\frac{\epsilon^{N-2}}{\left|x-x_{0}\right|^{N-2}}+\left(\frac{\epsilon}{\delta_{i}}\right)^{N-1} \frac{1}{\left|x-x_{0}\right|^{N-2}}+\delta_{i}^{2}+\left(\frac{\epsilon}{\delta_{i}}\right)^{N-2}\right], x \in \Omega_{\epsilon}
$$

for some positive constant $c$.

By (3.9) and (3.10), taking into account that the function $H\left(\cdot, x_{0}\right)$ is bounded in $\Omega_{\epsilon}$ we deduce that

$$
P_{\Omega_{\epsilon}} U_{\delta_{i}, \xi_{i}}(x)=U_{\delta_{i}, \xi_{i}}(x)+\bar{R}_{i_{\epsilon}}(x)
$$

where $\bar{R}_{i \epsilon}$ satisfies the pointwise estimate

$$
\left|\bar{R}_{i \epsilon}(x)\right| \leq c\left(\delta_{i}^{\frac{N-2}{2}}+\frac{\epsilon^{N-2}}{\delta_{i}^{\frac{N-2}{2}}} \frac{1}{\left|x-x_{0}\right|^{N-2}}\right), x \in \Omega_{\epsilon},
$$

A straightforward computation shows that

$$
\left\|\bar{R}_{i \epsilon}\right\|_{L^{\frac{2 N}{N-2}}\left(\Omega_{\epsilon}\right)} \rightarrow 0 \text { as } \epsilon \rightarrow 0,
$$

because of (3.4). Finally, we set

$$
R_{\epsilon}(x):=\sum_{i=1}^{k} \bar{R}_{i \epsilon}(x)+\psi_{\epsilon}(x), \quad x \in \Omega_{\epsilon}
$$

and by (3.7), (3.8), (3.11) and (3.13) we deduce (3.3) and (4.3).

The main result of this section is the following

Proposition 3.2. Let $\Omega_{\epsilon}:=\Omega \backslash B_{\epsilon}\left(x_{0}\right)$, where $\Omega$ is a smooth bounded domain in $\mathbb{R}^{N}$, $N \in \mathbb{N}, N \geq 3, x_{0} \in \Omega$ and $\epsilon>0$. Let $\phi_{\epsilon}$ be as in Proposition 3.1. There exists $\epsilon_{0}>0$ such that for any $\epsilon \in\left(0, \epsilon_{0}\right)$

$$
\int_{\Omega_{\epsilon}} \phi_{\epsilon} \varphi_{1, \epsilon} d x>0
$$

where $\varphi_{1, \epsilon}$ is the positive eigenfunction of the self-adjoint operator $L_{\epsilon}=-\Delta-p_{S}\left|\phi_{\epsilon}\right|^{p_{S}-1}$ on $L^{2}\left(\Omega_{\epsilon}\right)$ with domain $H^{2}\left(\Omega_{\epsilon}\right) \cap H_{0}^{1}\left(\Omega_{\epsilon}\right)$.

The proof of Proposition 3.2 relies on a spectral analysis of the linearized operator $-\Delta-p_{S}\left|\phi_{\epsilon}\right|^{p_{S}-1}$ for $\epsilon$ sufficiently small. By a suitable scaling, we can pass to the limit as $\epsilon$ goes to zero and study the analogous spectral problem on $\mathbb{R}^{N}$.

Before proving Proposition 3.2 we need some preliminary results.

In order to simplify the notation we define

$$
f(s):=|s|^{p_{S}-1} s, s \in \mathbb{R} .
$$


Moreover we set

$$
U:=U_{1,0}
$$

(see (3.1) for the definition of $U_{\delta, \xi}$ for any $\delta>0, \xi \in \mathbb{R}^{N}$ ).

Let us consider the linearization of the limit problem (3.2) around $U$, namely the linear problem

$$
\mathcal{L}^{*} v:=-\Delta v-f^{\prime}(U) v, v \in H^{1}\left(\mathbb{R}^{N}\right) .
$$

Let us define the first eigenvalue of $\mathcal{L}^{*}$ by

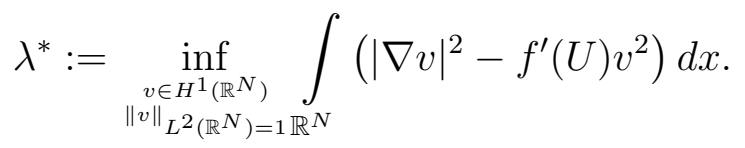

The following result holds true (see [13]).

Lemma 3.3. There hold true

(i) $\lambda^{*} \in(-\infty, 0)$,

(ii) there exists a unique positive minimizer $\varphi^{*}$ which is radial and radially nonincreasing. $\varphi^{*}$ is an eigenvector associated to $\lambda^{*}$,

(iii) every minimizing sequence has a subsequence which strongly converges in $L^{2}\left(\mathbb{R}^{N}\right)$.

Now, we fix an integer $k \geq 1$ and we consider the $k$-tower solution $\phi_{\epsilon}$ found in Lemma 4.1. We scale the solution around $\xi_{k_{\epsilon}}$ using the fastest concentration parameter $\delta_{k \epsilon}$, i.e. set

$$
\tilde{\phi}_{\epsilon}(x):=(-1)^{k} \delta_{k \epsilon}^{\frac{N-2}{2}} \phi_{\epsilon}\left(\delta_{k \epsilon} x+\xi_{k \epsilon}\right), x \in \tilde{\Omega}_{\epsilon}:=\frac{\Omega_{\epsilon}-\xi_{k_{\epsilon}}}{\delta_{k \epsilon}}=\left\{x \in \mathbb{R}^{N}: \delta_{k \epsilon} x+\xi_{k_{\epsilon}} \in \Omega_{\epsilon}\right\},
$$

This scaling allows to see only the last bubble as it is shown in the next lemma.

Lemma 3.4. It holds true that

$$
\tilde{\phi}_{\epsilon}(x)=U(x)+\rho_{\epsilon}(x)+\tilde{R}_{\epsilon}(x), x \in \tilde{\Omega}_{\epsilon}
$$

with $U$ as in (3.15),

$$
\left\|\rho_{\epsilon}\right\|_{L^{q}\left(\tilde{\Omega}_{\epsilon}\right)} \rightarrow 0 \text { for any } q>\frac{2 N}{N-2} \quad \text { and } \quad\left\|\tilde{R}_{\epsilon}\right\|_{L^{\frac{2 N}{N-2}\left(\tilde{\Omega}_{\epsilon}\right)}} \rightarrow 0 \quad \text { as } \epsilon \rightarrow 0 .
$$

Proof. We use (3.3) and we get $\tilde{\phi}_{\epsilon}(x)=U(x)+\rho_{\epsilon}(x)+\tilde{R}_{\epsilon}(x)$, where we set

$$
\rho_{\epsilon}(x):=\delta_{k \epsilon}^{\frac{N-2}{2}} \sum_{i=1}^{k-1}(-1)^{i+k} U_{\delta_{i \epsilon}, \xi_{i \epsilon}}\left(\delta_{k \epsilon} x+\xi_{k \epsilon}\right) \text { and } \tilde{R}_{\epsilon}(x):=(-1)^{k} \delta_{k \epsilon}^{\frac{N-2}{2}} R_{\epsilon}\left(\delta_{k \epsilon} x+\xi_{k \epsilon}\right) .
$$

It is immediate to check that

$$
\left\|\tilde{R}_{\epsilon}\right\|_{L^{\frac{2 N}{N-2}}\left(\tilde{\Omega}_{\epsilon}\right)}=\left\|R_{\epsilon}\right\|_{L^{\frac{2 N}{N-2}}\left(\Omega_{\epsilon}\right)} \rightarrow 0 \text { as } \epsilon \rightarrow 0
$$


because of (4.3). Moreover, we have

$$
\begin{aligned}
\int_{\tilde{\Omega}_{\epsilon}}\left|\rho_{\epsilon}(x)\right|^{q} d x & \leq c \sum_{i=1}^{k-1} \int_{\tilde{\Omega}_{\epsilon}}\left(\frac{\delta_{k}^{\frac{N-2}{2}} \delta_{i}^{\frac{N-2}{2}}}{\left(\delta_{i}^{2}+\left|\delta_{k} x+\xi_{k}-\xi_{i}\right|^{2}\right)^{\frac{N-2}{2}}}\right)^{q}\left(\text { setting } x=\frac{\delta_{i}}{\delta_{k}} y+\frac{\xi_{i}-\xi_{k}}{\delta_{k}}\right) \\
& =c \sum_{i=1}^{k-1}\left(\frac{\delta_{k}}{\delta_{i}}\right)^{\frac{N-2}{2} q-N} \int_{\frac{\Omega_{\epsilon}-\xi_{i}}{\delta_{i}}} \frac{1}{\left(1+|y|^{2}\right)^{\frac{N-2}{2} q}} d y\left(\text { we choose } \frac{q(N-2)}{2}>N\right) \\
& \left.\leq c \sum_{i=1}^{k-1}\left(\frac{\delta_{k}}{\delta_{i}}\right)^{\frac{N-2}{2} q-N} \int_{\mathbb{R}^{N}} \frac{1}{\left(1+|y|^{2}\right)^{\frac{N-2}{2} q}} d y \rightarrow 0 \text { as } \epsilon \rightarrow 0 \text {. (because of }(3.4)\right) .
\end{aligned}
$$

We consider the linearized operator at $\phi_{\epsilon}$, that is

$$
\mathcal{L}_{\epsilon} v:=-\Delta v-f^{\prime}\left(\phi_{\epsilon}\right) v, v \in H_{0}^{1}\left(\Omega_{\epsilon}\right) .
$$

Let $\lambda_{\epsilon}$ the first eigenvalue of $\mathcal{L}_{\epsilon}$ and $\varphi_{\epsilon}$ the corresponding positive eigenfunction normalized in $L^{2}\left(\Omega_{\epsilon}\right)$, i.e.

$$
-\Delta \varphi_{\epsilon}-f^{\prime}\left(\phi_{\epsilon}\right) \varphi_{\epsilon}=\lambda_{\epsilon} \varphi_{\epsilon} \text { in } \Omega_{\epsilon}, \varphi_{\epsilon}=0 \text { on } \partial \Omega_{\epsilon}, \quad \varphi_{\epsilon}>0 \text { in } \Omega_{\epsilon},\left\|\varphi_{\epsilon}\right\|_{L^{2}\left(\Omega_{\epsilon}\right)}=1 .
$$

Lemma 3.5. It holds true that $\lambda_{\epsilon}<0$.

Proof. It is enough to remark that by the definition of the first eigenvalue, i.e.

$$
\lambda_{\epsilon}:=\min _{\substack{v \in H_{0}^{1}\left(\Omega_{\epsilon}\right) \\\|v\|_{L^{2}\left(\Omega_{\epsilon}\right)}}} \int_{\Omega_{\epsilon}}\left(|\nabla v|^{2}-f^{\prime}\left(\phi_{\epsilon}\right) v^{2}\right) d x
$$

taking the solution $v_{\epsilon}:=\phi_{\epsilon} /\left\|\phi_{\epsilon}\right\|_{L^{2}\left(\Omega_{\epsilon}\right)}$ as a test function we get

$$
\lambda_{\epsilon} \leq \frac{\int_{\Omega_{\epsilon}}\left(\left|\nabla \phi_{\epsilon}\right|^{2}-f^{\prime}\left(\phi_{\epsilon}\right) \phi_{\epsilon}^{2}\right) d x}{\int_{\Omega_{\epsilon}} \phi_{\epsilon}^{2} d x}=\left(1-p_{S}\right) \frac{\int_{\Omega_{\epsilon}}\left|\phi_{\epsilon}\right|^{p_{S}+1} d x}{\int_{\Omega_{\epsilon}} \phi_{\epsilon}^{2} d x}<0 .
$$

Let us define

$$
\tilde{\varphi}_{\epsilon}(x):=\delta_{k \epsilon}^{\frac{N}{2}} \varphi_{\epsilon}\left(\delta_{k \epsilon} x+\xi_{k \epsilon}\right) \text { if } x \in \tilde{\Omega}_{\epsilon}, \tilde{\varphi}_{\epsilon}(x):=0 \text { if } x \notin \tilde{\Omega}_{\epsilon} \text { and } \tilde{\lambda}_{\epsilon}:=\delta_{k \epsilon}^{2} \lambda_{\epsilon} .
$$

Then, it is immediate to check that $\tilde{\varphi}_{\epsilon}$ solves

$$
-\Delta \tilde{\varphi}_{\epsilon}-f^{\prime}\left(\tilde{\phi}_{\epsilon}\right) \tilde{\varphi}_{\epsilon}=\tilde{\lambda}_{\epsilon} \tilde{\varphi}_{\epsilon} \text { in } \tilde{\Omega}_{\epsilon}, \tilde{\varphi}_{\epsilon}=0 \text { on } \partial \tilde{\Omega}_{\epsilon}, \quad \tilde{\varphi}_{\epsilon}>0 \text { in } \tilde{\Omega}_{\epsilon},\left\|\tilde{\varphi}_{\epsilon}\right\|_{L^{2}\left(\mathbb{R}^{n}\right)}=1 \text {. }
$$

Lemma 3.6. There hold true that

(i) There exists $c>0$ such that $\left\|\tilde{\varphi}_{\epsilon}\right\|_{H_{0}^{1}\left(\tilde{\Omega}_{\epsilon}\right)} \leq c$,

(ii) $\int_{\tilde{\Omega}_{\epsilon}}\left|f^{\prime}\left(\tilde{\phi}_{\epsilon}\right)-f^{\prime}(U)\right| \tilde{\varphi}_{\epsilon}^{2} d x \rightarrow 0$ as $\epsilon \rightarrow 0$. $\tilde{\Omega}_{\epsilon}$ 
Proof. We have

$$
\begin{aligned}
& \int_{\tilde{\Omega}_{\epsilon}}\left|\nabla \tilde{\varphi}_{\epsilon}(x)\right|^{2} d x=\int_{\tilde{\Omega}_{\epsilon}} f^{\prime}\left(\tilde{\phi}_{\epsilon}\right) \tilde{\varphi}_{\epsilon}^{2} d x+\tilde{\lambda}_{\epsilon} \int_{\tilde{\Omega}_{\epsilon}} \tilde{\varphi}_{\epsilon}^{2} d x \text { (we use that } \tilde{\lambda}_{\epsilon}<0 \text { because of Lemma 3.5) } \\
& \leq \int_{\tilde{\Omega}_{\epsilon}} f^{\prime}\left(\tilde{\phi}_{\epsilon}\right) \tilde{\varphi}_{\epsilon}^{2} d x=\int_{\tilde{\Omega}_{\epsilon}} f^{\prime}(U) \tilde{\varphi}_{\epsilon}^{2} d x+\int_{\tilde{\Omega}_{\epsilon}}\left(f^{\prime}\left(\tilde{\phi}_{\epsilon}\right)-f^{\prime}(U)\right) \tilde{\varphi}_{\epsilon}^{2} d x
\end{aligned}
$$

Now,

$$
\int_{\tilde{\Omega}_{\epsilon}} f^{\prime}(U) \tilde{\varphi}_{\epsilon}^{2} d x \leq\left\|f^{\prime}(U)\right\|_{L^{\infty}\left(\mathbb{R}^{N}\right)}\left\|\tilde{\varphi}_{\epsilon}\right\|_{L^{2}\left(\mathbb{R}^{N}\right)} \leq c
$$

for some positive constant $c$. Moreover it is easy to check that

$$
|| a+\left.b\right|^{\alpha-1}(a+b)-|a|^{\alpha-1} a \mid \leq\left\{\begin{array}{l}
c \min \left\{|a|^{\alpha-1}|b|,|b|^{\alpha}\right\} \forall a, b \in \mathbb{R}, \text { if } 0<\alpha \leq 1 \\
c\left(|a|^{\alpha-1}|b|+|b|^{\alpha}\right) \forall a, b \in \mathbb{R}, \text { if } \alpha>1,
\end{array}\right.
$$

where $c$ is a positive constant only depending on $\alpha$.

Hence by (3.22) with $\alpha=p_{S}-1$ we get

$$
\left|f^{\prime}\left(\tilde{\phi}_{\epsilon}\right)-f^{\prime}(U)\right| \leq\left\{\begin{array}{l}
c\left|\tilde{\phi}_{\epsilon}-U\right|^{p_{S}-1} \text { if } 1<p_{S} \leq 2 \\
c\left(|U|^{p_{S}-2}\left|\tilde{\phi}_{\epsilon}-U\right|+\left|\tilde{\phi}_{\epsilon}-U\right|^{p_{S}-1}\right) \quad \text { if } p_{S}>2
\end{array}\right.
$$

Then if $p_{S} \leq 2$, i.e. $N \geq 6$, we get

$$
\begin{aligned}
\int_{\tilde{\Omega}_{\epsilon}}\left|f^{\prime}\left(\tilde{\phi}_{\epsilon}\right)-f^{\prime}(U)\right| \tilde{\varphi}_{\epsilon}^{2} d x & \leq c \int_{\tilde{\Omega}_{\epsilon}}\left|\tilde{\phi}_{\epsilon}-U\right|^{p_{S}-1} \tilde{\varphi}_{\epsilon}^{2} d x \text { (we use Lemma 3.4) } \\
& \left.\leq c \int_{\tilde{\Omega}_{\epsilon}}\left(\left|\rho_{\epsilon}\right|^{p_{S}-1}+\left|\tilde{R}_{\epsilon}\right|^{p_{S}-1}\right) \tilde{\varphi}_{\epsilon}^{2} d x \text { (by Hölder's inequality with } t>N / 2\right) \\
& \leq c\left\|\rho_{\epsilon}\right\|_{L^{\left(p_{S}-1\right) t}\left(\tilde{\Omega}_{\epsilon}\right)}^{p_{S}-1}\left\|\tilde{\varphi}_{\epsilon}\right\|_{L^{\frac{2 t}{t-1}}\left(\tilde{\Omega}_{\epsilon}\right)}^{2}+c\left\|\tilde{R}_{\epsilon}\right\|_{L^{\frac{2 N}{N-2}\left(\tilde{\Omega}_{\epsilon}\right)}}^{p_{S}-1}\left\|\tilde{\varphi}_{\epsilon}\right\|_{L^{\frac{2 N}{N-2}}\left(\tilde{\Omega}_{\epsilon}\right)}^{2}
\end{aligned}
$$

and if $p_{S}>2$, i.e. $N=3,4,5$, we get

$$
\begin{aligned}
\int_{\tilde{\Omega}_{\epsilon}}\left|f^{\prime}\left(\tilde{\phi}_{\epsilon}\right)-f^{\prime}(U)\right| \tilde{\varphi}_{\epsilon}^{2} d x & \leq c \int_{\tilde{\Omega}_{\epsilon}}\left(|U|^{p_{S}-2}\left|\tilde{\phi}_{\epsilon}-U\right|+\left|\tilde{\phi}_{\epsilon}-U\right|^{p_{S}-1}\right) \tilde{\varphi}_{\epsilon}^{2} d x \text { (we use Lemma 3.4) } \\
& \leq c \int_{\tilde{\Omega}_{\epsilon}}\left[|U|^{p_{S}-2}\left(\left|\rho_{\epsilon}\right|+\left|\tilde{R}_{\epsilon}\right|\right)+\left|\rho_{\epsilon}\right|^{p_{S}-1}+\left|\tilde{R}_{\epsilon}\right|^{p_{S}-1}\right] \tilde{\varphi}_{\epsilon}^{2} d x
\end{aligned}
$$

(we apply Hölder's inequality with $t>N / 2$ )

$$
\begin{aligned}
\leq & c\|U\|_{L^{\infty}\left(\mathbb{R}^{N}\right)}^{p_{S}-2}\left\|\rho_{\epsilon}\right\|_{L^{t}\left(\tilde{\Omega}_{\epsilon}\right)}\left\|\tilde{\varphi}_{\epsilon}\right\|_{L^{\frac{2 t}{t-1}}\left(\tilde{\Omega}_{\epsilon}\right)}^{2} \\
& +c\|U\|_{L^{\frac{2 N}{6-N}\left(\tilde{\Omega}_{\epsilon}\right)}}^{p_{S}-2}\left\|\tilde{R}_{\epsilon}\right\|_{L^{\frac{2 N}{N-2}}\left(\tilde{\Omega}_{\epsilon}\right)}\left\|\tilde{\varphi}_{\epsilon}\right\|_{L^{\frac{2 N}{N-2}}\left(\tilde{\Omega}_{\epsilon}\right)}^{2} \\
& +c\left\|\rho_{\epsilon}\right\|_{L^{\left(p_{S}-1\right) t}\left(\tilde{\Omega}_{\epsilon}\right)}^{p-1}\left\|\tilde{\varphi}_{\epsilon}\right\|_{L^{\frac{2 t}{t-1}}\left(\tilde{\Omega}_{\epsilon}\right)}^{2}+c\left\|\tilde{R}_{\epsilon}\right\|_{L^{\frac{2 N}{N-2}}\left(\tilde{\Omega}_{\epsilon}\right)}^{p-1}\left\|\tilde{\varphi}_{\epsilon}\right\|_{L^{\frac{2 N}{N-2}}\left(\tilde{\Omega}_{\epsilon}\right)}^{2}
\end{aligned}
$$


Now, we remark that

$$
\frac{2 t}{t-1}=2 \theta+\frac{2 N}{N-2}(1-\theta) \quad \text { with } \theta:=\frac{2 t-N}{2(t-1)} \in(0,1)
$$

and so by interpolation

$$
\left\|\tilde{\varphi}_{\epsilon}\right\|_{L^{\frac{2 t}{t-1}\left(\tilde{\Omega}_{\epsilon}\right)}} \leq\left\|\tilde{\varphi}_{\epsilon}\right\|_{L^{2}\left(\tilde{\Omega}_{\epsilon}\right)}^{\frac{\theta(t-1)}{t}}\left\|\tilde{\varphi}_{\epsilon}\right\|_{L^{\frac{2 N}{N-2}\left(\tilde{\Omega}_{\epsilon}\right)}}^{\frac{N}{2 t}} \leq\left\|\tilde{\varphi}_{\epsilon}\right\|_{L^{\frac{2 N}{N-2}}\left(\tilde{\Omega}_{\epsilon}\right)}^{\frac{N}{2 t}}
$$

because $\left\|\tilde{\varphi}_{\epsilon}\right\|_{L^{2}\left(\tilde{\Omega}_{\epsilon}\right)} \leq 1$. Finally, we collect (3.20)-(3.25), we use Sobolev's inequality $\left\|\tilde{\varphi}_{\epsilon}\right\|_{L^{\frac{2 N}{N-2}}\left(\tilde{\Omega}_{\epsilon}\right)} \leq$ $c\left\|\tilde{\varphi}_{\epsilon}\right\|_{H_{0}^{1}\left(\tilde{\Omega}_{\epsilon}\right)}$, we also estimate (3.17) and we get

$$
(1-\alpha(\epsilon))\left\|\tilde{\varphi}_{\epsilon}\right\|_{H_{0}^{1}\left(\tilde{\Omega}_{\epsilon}\right)}^{2} \leq c+\beta(\epsilon)\left\|\tilde{\varphi}_{\epsilon}\right\|_{H_{0}^{1}\left(\tilde{\Omega}_{\epsilon}\right)}^{\frac{N}{t}}
$$

where $\alpha(\epsilon), \beta(\epsilon) \rightarrow 0$ as $\epsilon \rightarrow 0$. Therefore (i) follows, because $t>N / 2$.

By (i), by estimates (3.23), (3.24) and (3.25) and by (3.17) we immediately get (ii).

Lemma 3.7. It holds true that

(i) $\lim _{\epsilon \rightarrow 0} \tilde{\lambda}_{\epsilon}=\lambda^{*}$

(ii) $\varphi_{\epsilon}$ strongly converges to $\varphi^{*}$ in $L^{2}\left(\mathbb{R}^{N}\right)$ as $\epsilon \rightarrow 0$.

Proof. Let us prove (i). By the definiton of $\lambda^{*}$ and by (3.19), we get

$$
\begin{aligned}
\lambda^{*} & \leq \int_{\mathbb{R}^{N}}\left(\left|\nabla \tilde{\varphi}_{\epsilon}\right|^{2}-f^{\prime}(U) \tilde{\varphi}_{\epsilon}^{2}\right) d x=\int_{\tilde{\Omega}_{\epsilon}}\left(\left|\nabla \tilde{\varphi}_{\epsilon}\right|^{2}-f^{\prime}(U) \tilde{\varphi}_{\epsilon}^{2}\right) d x \\
& =\int_{\tilde{\Omega}_{\epsilon}}\left(\left|\nabla \tilde{\varphi}_{\epsilon}\right|^{2}-f^{\prime}\left(\tilde{\phi}_{\epsilon}\right) \tilde{\varphi}_{\epsilon}^{2}\right) d x+\int_{\tilde{\Omega}_{\epsilon}}\left(f^{\prime}\left(\tilde{\phi}_{\epsilon}\right)-f^{\prime}(U)\right) \tilde{\varphi}_{\epsilon}^{2} d x \\
& =\tilde{\lambda}_{\epsilon}+\alpha(\epsilon), \text { where } \alpha(\epsilon) \rightarrow 0 \text { as } \epsilon \rightarrow 0,
\end{aligned}
$$

because of (ii) of Lemma 3.6.

On the other hand, let us consider a regular cut-off function $\chi_{\epsilon}(x)=\chi_{\epsilon}\left(\left|x-x_{0}\right|\right)$ such that $0 \leq \chi_{\epsilon} \leq 1$ and

$$
\chi_{\epsilon}(r)=1 \text { if } 4 \epsilon \leq r \leq \frac{\operatorname{diam}(\Omega)}{4} \text { and } \chi_{\epsilon}(|x|)=0 \text { if } r \leq 2 \epsilon \text { or } r \geq \frac{\operatorname{diam}(\Omega)}{2}
$$

Let us consider the functions

$w_{\epsilon}(x):=\frac{\chi_{\epsilon}(x) \varphi^{*}\left(\left(x-\xi_{k_{\epsilon}}\right) / \delta_{k_{\epsilon}}\right)}{\left\|\chi_{\epsilon}(x) \varphi^{*}\left(\left(x-\xi_{k_{\epsilon}}\right) / \delta_{k_{\epsilon}}\right)\right\|_{L^{2}\left(\Omega_{\epsilon}\right)}}, x \in \Omega_{\epsilon}$ and $\tilde{w}_{\epsilon}(y):=\frac{\chi_{\epsilon}\left(\delta_{k_{\epsilon}} y+\xi_{k_{\epsilon}}\right) \varphi^{*}(y)}{\left\|\chi_{\epsilon}\left(\delta_{k \epsilon} y+\xi_{k_{\epsilon}}\right) \varphi^{*}(y)\right\|_{L^{2}\left(\tilde{\Omega}_{\epsilon}\right)}}, y \in \tilde{\Omega}_{\epsilon}$.

It is easy to check that

$$
\tilde{w}_{\epsilon} \rightarrow \varphi^{*} \text { in } H^{1}\left(\mathbb{R}^{N}\right) \text { as } \epsilon \rightarrow 0 .
$$

By the definition of $\lambda_{\epsilon}$ and scaling $x=\delta_{k \epsilon} y+\xi_{k_{\epsilon}}$ we get

$$
\lambda_{\epsilon} \leq \int_{\Omega_{\epsilon}}\left(\left|\nabla w_{\epsilon}\right|^{2}-f^{\prime}\left(\phi_{\epsilon}\right) w_{\epsilon}^{2}\right) d x=\frac{1}{\delta_{k_{\epsilon}}^{2}} \int_{\tilde{\Omega}_{\epsilon}}\left(\left|\nabla \tilde{w}_{\epsilon}\right|^{2}-f^{\prime}\left(\tilde{\phi}_{\epsilon}\right) \tilde{w}_{\epsilon}^{2}\right) d y
$$


which implies

$$
\begin{aligned}
\tilde{\lambda}_{\epsilon} & \leq \int_{\tilde{\Omega}_{\epsilon}}\left(\left|\nabla \tilde{w}_{\epsilon}\right|^{2}-f^{\prime}\left(\tilde{\phi}_{\epsilon}\right) \tilde{w}_{\epsilon}^{2}\right) d y=\int_{\mathbb{R}^{N}}\left(\left|\nabla \tilde{w}_{\epsilon}\right|^{2}-f^{\prime}\left(\tilde{\phi}_{\epsilon}\right) \tilde{w}_{\epsilon}^{2}\right) d y \\
& =\int_{\mathbb{R}^{N}}\left(\left|\nabla \tilde{w}_{\epsilon}\right|^{2}-f^{\prime}(U) \tilde{w}_{\epsilon}^{2}\right) d y+\int_{\mathbb{R}^{n}}\left(f^{\prime}(U)-f^{\prime}\left(\tilde{\phi}_{\epsilon}\right)\right) \tilde{w}_{\epsilon}^{2} d y \\
& =\lambda^{*}+\beta(\epsilon), \text { where } \beta(\epsilon) \rightarrow 0 \text { as } \epsilon \rightarrow 0,
\end{aligned}
$$

because by (3.27) we deduce that

$$
\int_{\mathbb{R}^{N}}\left(\left|\nabla \tilde{w}_{\epsilon}\right|^{2}-f^{\prime}(U) \tilde{w}_{\epsilon}^{2}\right) d y \rightarrow \int_{\mathbb{R}^{N}}\left(\left|\nabla \varphi^{*}\right|^{2}-f^{\prime}(U) \varphi^{* 2}\right) d y=\lambda^{*} \text { as } \epsilon \rightarrow 0
$$

and arguing exactly as in the proof of (ii) of Lemma 3.6 we get

$$
\int_{\mathbb{R}^{n}}\left(f^{\prime}(U)-f^{\prime}\left(\tilde{\phi}_{\epsilon}\right)\right) \tilde{w}_{\epsilon}^{2} d y \rightarrow 0 \text { as } \epsilon \rightarrow 0 .
$$

Finally, by (3.26) and (3.28) the claim follows.

Next we prove (ii). By the definition of $\tilde{\lambda}_{\epsilon}$ and (i) we have

$$
\int_{\widetilde{\Omega}_{\epsilon}}\left(\mid \nabla \tilde{\varphi}_{\epsilon} \|^{2}-f^{\prime}\left(\tilde{\phi}_{\epsilon}\right) \tilde{\varphi}_{\epsilon}^{2}\right) d y=\tilde{\lambda}_{\epsilon} \rightarrow \lambda^{*} \text { as } \epsilon \rightarrow 0
$$

which implies that $\tilde{\varphi}_{\epsilon}$ is a minimizing sequence for (3.16) and so the claim follows by Lemma 3.3 .

Proof of Proposition 3.2. We now prove that

$$
\liminf _{\epsilon \rightarrow 0} \int_{\Omega_{\epsilon}} \phi_{\epsilon} \varphi_{\epsilon} d x>0 .
$$

We multiply equation (1.4) by $\varphi_{\epsilon}$ and equation (3.18) by $\phi_{\epsilon}$, we subtract the two equations and we get

$$
\int_{\Omega_{\epsilon}} \phi_{\epsilon} \varphi_{\epsilon} d x=-\frac{p_{S}-1}{\lambda_{\epsilon}} \int_{\Omega_{\epsilon}} f\left(\phi_{\epsilon}\right) \varphi_{\epsilon} d x
$$

Therefore, we are lead to study the sign of the right hand side of (3.29). We are going to prove that

$$
\lim _{\epsilon \rightarrow 0} \delta_{k \epsilon} \int_{\Omega_{\epsilon}} f\left(\phi_{\epsilon}\right) \varphi_{\epsilon} d x=\int_{\mathbb{R}^{N}} f(U) \varphi^{*} d x
$$


Since the right hand side of (3.30) is positive, this will imply that the right hand side of (3.29) is positive and finally the claim will follow.

Let us prove (3.30). We have

$$
\begin{aligned}
\delta_{k \epsilon} \int_{\Omega_{\epsilon}} f\left(\phi_{\epsilon}\right) \varphi_{\epsilon} d x-\int_{\mathbb{R}^{N}} f(U) \varphi^{*} d x & =\int_{\tilde{\Omega}_{\epsilon}} f\left(\tilde{\phi}_{\epsilon}\right) \tilde{\varphi}_{\epsilon} d x-\int_{\mathbb{R}^{N}} f(U) \varphi^{*} d x \\
& =\int_{\tilde{\Omega}_{\epsilon}}\left[f\left(\tilde{\phi}_{\epsilon}\right)-f(U)\right] \tilde{\varphi}_{\epsilon} d x+\int_{\mathbb{R}^{N}} f(U)\left[\tilde{\varphi}_{\epsilon}-\varphi^{*}\right] d x .
\end{aligned}
$$

By Hölder's inequality we get

$$
\left|\int_{\mathbb{R}^{N}} f(U)\left[\tilde{\varphi}_{\epsilon}-\varphi^{*}\right] d x\right| \leq\|f(U)\|_{L^{2}\left(\mathbb{R}^{N}\right)}\left\|\tilde{\varphi}_{\epsilon}-\varphi^{*}\right\|_{L^{2}\left(\mathbb{R}^{N}\right)} \rightarrow 0 \text { as } \epsilon \rightarrow 0
$$

because of (ii) of Lemma 3.7. Moreover, by (3.22) with $\alpha=p_{S}$ and using Hölder's inequality we get (here we choose $q>\frac{2 N}{N-2}$ with $q(N-8)<2 N$ )

$$
\begin{aligned}
\left|\int_{\tilde{\Omega}_{\epsilon}}\left[f\left(\tilde{\phi}_{\epsilon}\right)-f(U)\right] \tilde{\varphi}_{\epsilon} d x\right| \leq & c \int_{\tilde{\Omega}_{\epsilon}}\left(f^{\prime}(U)\left|\tilde{\phi}_{\epsilon}-U\right|+\left|\tilde{\phi}_{\epsilon}-U\right|^{p_{S}}\right)\left|\tilde{\varphi}_{\epsilon}\right| d x \\
\leq & c \int_{\tilde{\Omega}_{\epsilon}}\left[U^{\frac{4}{N-2}}\left(\left|\rho_{\epsilon}\right|+\left|\tilde{R}_{\epsilon}\right|\right)+\left(\left|\rho_{\epsilon}\right|^{\frac{N+2}{N-2}}+\left|\tilde{R}_{\epsilon}\right|^{\frac{N+2}{N-2}}\right)\right]\left|\tilde{\varphi}_{\epsilon}\right| d x \\
\leq & c\|\|_{L^{\frac{8 q}{(q-2)(N-2)}}\left(\mathbb{R}^{N}\right)}^{\frac{4}{N-2}}\left\|\rho_{\epsilon}\right\|_{L^{q}\left(\tilde{\Omega}_{\epsilon}\right)}\left\|\tilde{\varphi}_{\epsilon}\right\|_{L^{2}\left(\tilde{\Omega}_{\epsilon}\right)} \\
& +c\|\|_{L^{\frac{2 N}{N-2}}\left(\mathbb{R}^{N}\right)}^{\frac{4}{N-2}}\left\|\tilde{R}_{\epsilon}\right\|_{L^{\frac{2 N}{N-2}}\left(\tilde{\Omega}_{\epsilon}\right)}\left\|\tilde{\varphi}_{\epsilon}\right\|_{L^{\frac{2 N}{N-2}}\left(\tilde{\Omega}_{\epsilon}\right)} \\
& +c\left\|\rho_{\epsilon}\right\|_{L^{\frac{2(N+2)}{N-2}}\left(\tilde{\Omega}_{\epsilon}\right)}^{\frac{N+2}{N-2}}\left\|\tilde{\varphi}_{\epsilon}\right\|_{L^{2}\left(\tilde{\Omega}_{\epsilon}\right)}+c\left\|\tilde{R}_{\epsilon}\right\|_{L^{\frac{N+2}{N-2}}\left(\tilde{\Omega}_{\epsilon}\right)}^{\frac{2+2}{N-2}}\left\|\tilde{\varphi}_{\epsilon}\right\|_{L^{\frac{2 N}{N-2}}\left(\tilde{\Omega}_{\epsilon}\right)}
\end{aligned}
$$

(we apply Lemma 3.6 and estimate (3.17)

$$
\leq \alpha(\epsilon)
$$

where $\alpha(\epsilon) \rightarrow 0$ as $\epsilon \rightarrow 0$. Therefore the claim follows.

\section{Proof of Theorem 1.2}

The proof of Theorem 1.2 consists in finding a radial sign changing stationary solution to (1.4) which satisfies (2.5). Then Proposition 2.2 applies and the conclusion follows from the last statement of Proposition 2.1.

As the radial sign changing stationary solution we take now a radial $k$-tower solution, which exists in the annulus with a sufficiently small hole, as next result asserts:

Lemma 4.1 (Existence of radial $k$-tower stationary solutions). Let $\Omega_{\epsilon}:=\left\{x \in \mathbb{R}^{N}: 0<\right.$ $\epsilon<|x|<1\}, N \in \mathbb{N}, N \geq 3$ and $\epsilon>0$ small. For any integer $k \geq 2$ there exists $\epsilon_{k}>0$ such 
that for any $\epsilon \in\left(0, \epsilon_{k}\right)$ problem (1.4) has a radial $k$-tower sign-changing stationary solution $\widehat{\phi}_{\epsilon}$ whose profile is

$$
\widehat{\phi}_{\epsilon}(x)=\sum_{i=1}^{k}(-1)^{i} U_{\delta_{i_{\epsilon}}, 0}(x)+\widehat{R}_{\epsilon}(x), x \in \Omega_{\epsilon}
$$

where the concentration parameters $\delta_{i \epsilon}$ 's satisfy

$$
\delta_{i \epsilon}:=\widehat{d}_{i \epsilon} \epsilon^{\frac{2 i-1}{2 k}}, \widehat{d}_{i \epsilon} \in \mathbb{R} \quad \text { and } \quad \widehat{d}_{i_{\epsilon}} \rightarrow \widehat{d}_{i}>0 \text { as } \epsilon \rightarrow 0 \quad \text { for } i=1, \ldots, k,
$$

and the remainder term $\widehat{R_{\epsilon}}$ is radial and satisfies

$$
\left\|\widehat{R}_{\epsilon}\right\|_{L^{\frac{2 N}{N-2}}\left(\Omega_{\epsilon}\right)} \rightarrow 0 \text { as } \epsilon \rightarrow 0 .
$$

Proof. Combining the ideas in [14] with the general arguments in [9] one can prove that for any integer $k \geq 1$ there exists $\epsilon_{k}>0$ such that for any $\epsilon \in\left(0, \epsilon_{k}\right)$ problem (1.4) has a stationary solution $\widehat{\phi}_{\epsilon}$ whose profile is

$$
\widehat{\phi}_{\epsilon}(x)=\sum_{i=1}^{k}(-1)^{i} P_{\Omega_{\epsilon}} U_{\delta_{i \epsilon}, 0}(x)+\widehat{\psi}_{\epsilon}(x), x \in \Omega_{\epsilon}
$$

where the concentration parameters $\delta_{1}=\delta_{1 \epsilon}, \ldots, \delta_{k}=\delta_{k \epsilon}$ satisfy (4.2) and the remainder term $\widehat{\psi}_{\epsilon}$ is radial and satisfies

$$
\left\|\widehat{\psi}_{\epsilon}\right\|_{H_{0}^{1}\left(\Omega_{\epsilon}\right)} \rightarrow 0 \text { as } \epsilon \rightarrow 0 .
$$

The conclusion follows similarly as in the proof of Lemma 3.1.

Performing an asymptotic spectral analysis similarly as in the proof of Proposition 3.2 we can now prove the following result and hence conclude

Proposition 4.2. Let $\Omega_{\epsilon}:=\left\{x \in \mathbb{R}^{N}: 0<\epsilon<|x|<1\right\}, N \in \mathbb{N}, N \geq 3$ and $\epsilon>0$. Let $\widehat{\phi}_{\epsilon}$ be as in Proposition 4.1. There exists $\epsilon_{0}>0$ such that for any $\epsilon \in\left(0, \epsilon_{0}\right)$

$$
\int_{\Omega_{\epsilon}} \widehat{\phi}_{\epsilon} \varphi_{1, \epsilon} d x>0
$$

where $\varphi_{1, \epsilon}$ is the positive eigenfunction of the self-adjoint operator $L_{\epsilon}=-\Delta-p_{S}\left|\widehat{\phi}_{\epsilon}\right|^{p_{S}-1}$ on $L^{2}\left(\Omega_{\epsilon}\right)$ with domain $H^{2}\left(\Omega_{\epsilon}\right) \cap H_{0}^{1}\left(\Omega_{\epsilon}\right)$.

\section{ACKNOWLEDGMENTS}

Portions of this research were done while the first author was visiting the second one. The first author would like to thank the Facultad de Matemáticas of the Pontificia Universidad Católica de Chile for the warm hospitality during her staying. The authors would like to express their gratitude to Thierry Cazenave for many helpful comments and remarks. 


\section{REFERENCES}

[1] A. Bahri, J.-M. Coron, On a nonlinear elliptic equation involving the critical Sobolev exponent: the effect of the topology of the domain, Comm. Pure Appl. Math. 41 (1988), 253-294.

[2] T. Cazenave, F. Dickstein, F.B. Weissler, Sign-changing stationary solutions and blow up for the nonlinear heat equation in a ball, Math. Ann. 344 (2) (2009), 431-449.

[3] J.-M. Coron, Topologie et cas limite des injections de Sobolev, C.R. Acad. Sc. Paris 299 s.I (1984), 209-212.

[4] F. De Marchis, I. Ianni, Blow up of solutions of semilinear heat equations in non radial domains of $\mathbb{R}^{2}$, preprint (2014) (arXiv:1403.0115).

[5] F. Dickstein, F. Pacella, B. Sciunzi, Sign-changing stationary solutions and blow up for the nonlinear heat equation in dimension two, preprint (2013) (arXiv:1304.2571).

[6] V.A. Galaktionov, J.R. King, Composite structure of global unbounded solutions of nonlinear heat equations with critical Sobolev exponents, J. Differential Equations 189 (2003), 199-233.

[7] V.A. Galaktionov, J.L. Vazquez, Continuation of blowup solutions of nonlinear heat equations in several space dimensions, Comm. Pure Appl. Math. 50 (1997), 1-67.

[8] F. Gazzola, T. Weth, Finite-time blow-up and global solutions for semilinear parabolic equations with initial data at high energy levels, Differ. Integral Equ. 8 (9) (2005), 961-990.

[9] Y. Ge, M. Musso, A. Pistoia, Sign Changing Tower of Bubbles for an Elliptic Problem at the Critical Exponent in Pierced Non-Symmetric Domains, , Comm. PDE 35 (2010), 1-39.

[10] M. Ishiwata, On bounds for global solutions of semilinear parabolic equations with critical and subcritical Sobolev exponent, Differ. Integral Equ. 20 (9) (2007), 1021-1034.

[11] J.L. Kazdan, F.W. Warner, Remarks on some quasilinear elliptic equations, Comm. Pure Appl. Math. 28 (1975), 567-597.

[12] P.-L. Lions, Asymptotic behavior of some nonlinear heat equations, Nonlinear Phenomena Phys. D 5 (1982), 293-306.

[13] V. Marino, F. Pacella, B. Sciunzi, Blow up of solutions of semilinear heat equations in general domains Comm. in Contemp. Math., doi: 10.1142/S0219199713500429.

[14] M. Musso, A. Pistoia, Sign changing solutions to a nonlinear elliptic problem involving the critical Sobolev exponent in pierced domains, J. Math. Pures Appl. 86 (2006), 510-528.

[15] W.M. Ni, P.E. Sacks, J. Tavantzis, On the asymptotic behavior of solutions of certain quasilinear parabolic equations, J. Diff. Eqs. 54 (1984), 97-120.

[16] S.I. Pohozaev, Eigenfunctions of the equation $\Delta u+\lambda f(u)=0$, Soviet Math. Dokl. 5 (1965), 1408-1411.

[17] P. Quittner, P. Souplet, Superlinear parabolic problems, Birkhäuser Basel-Boston-Berlin 2007.

[18] T. Suzuki, Semilinear parabolic equation on bounded domain with critical Sobolev exponent, Indiana Univ. Math. J. 57 (7) (2008), 3365-3396.

(Isabella Ianni) Dipartimento di Matematica e Fisica, Seconda Università degli Studi di Napoli, Viale Lincoln 5, 81100 Caserta, italy

E-mail address: isabella.ianni@unina2.it

(Monica Musso) Departamento de Matematicas, Pontificia Universidad Católica de Chile Casilla 306, Correo 22 Santiago, Chile.

E-mail address: mmusso@mat.puc.cl

(Angela Pistoia) Dipartimento SBAi, Università di Roma "La Sapienza", via Antonio Scarpa 16, 00161 Roma, Italy

E-mail address: pistoia@dmmm.uniroma1.it 